\title{
Serial attention within working memory
}

\author{
HUGH GARAVAN \\ Bowling Green State University, Bowling Green, Ohio
}

\begin{abstract}
It is proposed that people are limited to attending to just one "object" in working memory (WM) at any one time. Consequently, many cognitive tasks, and much of everyday thought, necessitate switches between WM items. The research to be presented measured the time involved in switching attention between objects in WM and sought to elaborate the processes underlying such switches. Two experiments required subjects to maintain two running counts; the order in which the counts were updated necessitated frequent switches between them. Even after intensive practice, a time cost was incurred when subjects updated the two counts in succession, relative to updating the same count twice. This time cost was interpreted as being due to a distinct switching mechanism that controls an internal focus of attention large enough for just one object (count) at a time. This internal focus of attention is a subset of WM (Cowan, 1988). Alternative visual and conceptual repetition-priming and memory retrieval explanations for the cost involved in switching between items in WM are addressed.
\end{abstract}

The issue of human attention and its processing capacity and limitations is an old one in psychology. William James claimed that we could focus our attention on just one "object" at a time. Though that object may in fact be a connected system of other objects, nevertheless, "they can only be known in a single pulse of consciousness for which they form one complex "object" (James, 1890, Vol. 1, p. 405). Pillsbury, discussing visual attention, continues in this theme: "It has long been a dogma of common sense, and was an accepted principle of the old rational psychology, that man can attend to but one thing at a time, that no more than a single impression can occupy the centre of consciousness at any given instant" (Pillsbury, 1908/1973, p. 64).

The present study investigated a similar limitation with regard to one's ability to attend to just one item in working memory. The purpose was to demonstrate that there exists a distinct attentional process that requires time to complete switches within working memory. This study provides an estimate of this switching parameter and considers the implications of such a limitation.

\section{Attention Switching}

Today, the same issues that James and Pillsbury were addressing motivate a sizable field of investigation. Studies of attention switching include switches between perceptual stimuli (Guzy \& Axelrod, 1972; Kerr, 1973; Mewhort, Thio, \& Birkenmayer, 1971; ten Hoopen \& Vos,

This PhD dissertation research was conducted at Bowling Green State University under the supervision of Michael E. Doherty, who provided substantial contributions to the ideas and tasks described herein. I also gratefully acknowledge comments by Rich Anderson, Nelson Cowan, Alice Healy, and an anonymous reviewer. Correspondence concerning this article should be addressed to H. Garavan, MFRC, Medical College of Wisconsin, 8701 Watertown Plank Rd., Milwaukee, WI 53226 (e-mail: hgaravan@mcw.edu).

-Accepted by previous editor, Geoffrey R. Loftus
1981), switches between perceptual stimuli and memorized lists (Carlson, Wenger, \& Sullivan, 1993; Dark, 1990; Weber, Burt, \& Noll, 1986), and switches between mental or task sets (Jersild, 1927; Laabs \& Stager, 1976; Rogers \& Monsell, 1995; Spector \& Biederman, 1976).

One of the classic paradigms for addressing attention switching within working memory is the Sternberg task (Sternberg, 1966, 1967, 1969). In Sternberg's studies, subjects were required to store a number of items in memory. They were then presented with a test item that could either be a member of the stored set, thus requiring a positive response, or not, requiring a negative response. Sternberg showed that reaction times (RTs) increased linearly with the size of the stored set, both for positive and for negative responses. Significantly, the positive and negative responses were equally affected by the memory set size, having similar intercepts and slopes. There was also no serial position effect in which RTs might be affected by the location of a positive test item in the memory set. From these findings, Sternberg proposed a model of exhaustive serial comparisons in which each test item is compared with each individual item in the memory set until all comparisons have been made. If a match has been found, a positive response is made; if not, there is a negative response. Adopting such a seemingly inefficient strategy can be understood if one assumes that the determination of a match after each individual comparison would consume more time than would exhausting the set and making just one such determination.

For the present enquiry the Sternberg studies are of importance, as they demonstrated seriality in processing items in working memory (or active memory, to use Sternberg's term). They revealed that we do not have simultaneous and immediate access to all of the items currently in working memory. Instead, the serial comparison of items in working memory required switches between these items. One may assume that the comparisons did occur in working memory, as many of Stern- 
berg's experiments presented a new memory set just seconds before each trial (see, e.g., Sternberg, 1966, Experiment 1). In this particular experiment, each serial comparison required approximately $40 \mathrm{msec}$. This estimate appeared quite robust, remaining essentially unchanged for positive and negative trials (Sternberg, 1966), for degraded and intact stimuli (Sternberg, 1967), for nonsense forms and photographs of faces $(45 \mathrm{msec}$ and $56 \mathrm{msec}$, respectively; Sternberg, 1969), for unfamiliar and welllearned lists, and for different amounts of practice (Sternberg, 1967). As Sternberg acknowledged, the comparison time estimate contained both a comparison component and a switching component. His procedure would not allow their separate measurement, and he assumed that the time for each switch operation was independent of list length.

It should be noted that other memory-scanning experiments have found results incompatible with Sternberg's serial exhaustive search model. These include serial position effects (Corballis, Kirby, \& Miller, 1972), repetition effects (Baddeley \& Ecob, 1973), nonlinear set size effects (Briggs, 1974), and stimulus probability effects (Theois, Smith, Haviland, Traupmann, \& Moy, 1973). Alternative parallel models (see, e.g., Ratcliff, 1978) interpret the increase in RTs as the set size increases as being a consequence of having to distribute limited resources among a greater number of items. Ratcliff proposed that all items in the memory set, and perhaps all items in memory, are available and accessed in parallel. Indeed, some commentators suggest that parallel models appear to provide more satisfactory accounts of the entire range of phenomena associated with the Sternberg task (Greene, 1992).

Schneider and Shiffrin, however, also concluded in favor of a model of serial searches, after their investigations of memory scanning and automaticity (Schneider \& Shiffrin, 1977; Shiffrin \& Schneider, 1977). Their tasks also provided an estimate of the time required to switch between items in working memory. In these tasks, the subjects memorized a number of characters and then searched for any one of these characters in a series of rapidly presented visual displays. The displays contained a number of other characters that served as distractors. For Schneider and Shiffrin's purposes, a key manipulation was the relationship between the set of characters from which the memorized items were selected and the set of characters used as the distractors. Specifically, in the varied mapping condition, any one character could serve as either a memory item or a distractor item in different trials, whereas, in the consistent mapping condition, memory and distractor items never varied.

It is the data from the varied mapping condition that are of most interest for present purposes. For the data of this condition, Schneider and Shiffrin constructed a model in which the subjects compared each memory item in turn against all the visually displayed items, and terminated the search on discovering a match. Having compared a memory item with the visually displayed items and finding no match, the subjects then switched to the next memory item. The authors arrived at a measure of $42 \mathrm{msec}$ with highly practiced subjects for this switching operation.

In interpreting the switching costs of the previous studies, one is left unsure of how best to characterize the particular process(es) involved. These switching costs may reflect the operation of a distinct attention-switching mechanism or, alternatively, they may reflect the time for retrieving the next item in the memory set. These are, of course, difficult processes to empirically tease apartperhaps especially so, if one's focus is on switching between items in working memory. Nonetheless, this is an issue to which we will return later.

\section{The Present Study}

The present study focuses on the dynamics involved in switching attention between the same few items in working memory. It is important to note that the focus is on the switching of attention between what might be called objects of thought - that is, distinct representations in working memory. Motivating this investigation is the question: Are we capable of maintaining and attending to two distinct memories or concepts simultaneously? If not, then attending to two such items should require switches back and forth between the two. If this is the case, how long do such switches take, and what can we tell about the process(es) involved in such switches? For these purposes, the present study employed a dual-count task, described in detail below, in which subjects were required to keep two running counts in working memory. These counts were the objects of thought residing in working memory between which subjects must switch.

To convey a sense of the phenomenon of interest, the reader's attention is directed to Figure 1. Figure 1 contains a random scattering of circles and triangles. The task for the reader is to count how many of each are presented; the reader is encouraged to complete this task before proceeding.

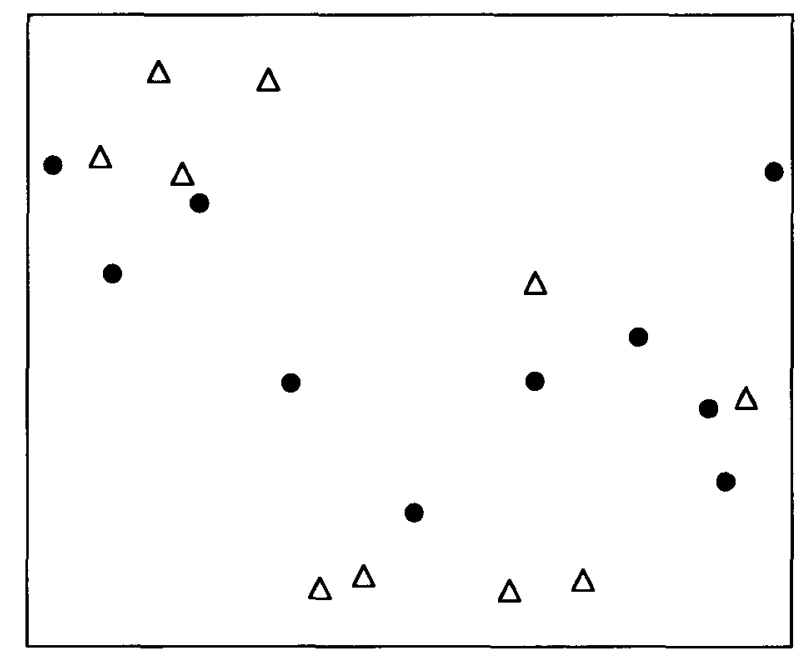

Figure 1. How many circles and triangles are there? 
There are, in fact, 10 circles and 10 triangles. My prediction is that readers did one of two things: they first counted the number of circles and then the number of triangles, or they first counted the number of triangles and then the number of circles. Probably very few, if any, readers counted both circles and triangles together-for example, by starting at a corner and updating two running counts while moving through the figure. Why should this be so? The hypothesis is that people can focus on just one item in working memory at a time. Maintaining two running counts thus requires switches between these counts, and this switching is effortful and therefore avoided. If the reader returns to Figure 1 and counts the circles and triangles together with two running counts, as described above, the reader will note that switching between counts is more difficult than updating the same count in succession. A task that requires maintaining two or more distinct concepts will, of necessity, evoke an effortful internal mechanism to switch between them.

This proposal is compatible with the hierarchical conceptualization of short-term memory (STM) described by Cowan $(1988,1993)$. This conceives of STM as an activated subset of long-term memory. One's current awareness and focus of attention is, in turn, a subset of one's STM. The remaining contents of STM are considered especially available (Cowan, 1993, p. 162), should one wish to shift one's focus to them. In this sense, though both counts are considered available, we can focus on only one at any one time.

\section{EXPERIMENT 1}

The subjects were asked to keep a count, without the use of external aids, of the number of two types of objects, presented one object at a time. Two types of geometric figures (two rectangles and two triangles) were presented on a computer screen. The subject's task was to maintain a count for each type of figure. Presentation of the figures was self-paced; a barpress response by the subject cleared the screen of the current figure and called up the next. Two types of count sequence were identified: a stimulus switch (SS), in which the subjects had to switch from one count to the other (e.g., if a rectangle followed a triangle or a triangle followed a rectangle), and a stimulus no switch (SNS), in which the subjects had to update the same count twice in a row (e.g., if a rectangle followed a rectangle or a triangle followed a triangle). Slower RTs for an SS relative to an SNS would be interpreted as evidence for the existence of an internal switching mechanism. The difference between the SS and the SNS RTs would provide an estimate of the mechanism's operation time.

\footnotetext{
Method

Subjects. The subjects were 10 students drawn from an introductory psychology subject pool. There were no special criteria for inclusion in this study. The experiment required two sessions, each
}

$1 \mathrm{~h}$ long, held at the same time of day on 2 consecutive days. Subjects received credit in partial fulfillment of their course requirements for participation.

Apparatus. A Macintosh SE computer and SuperLab, a general purpose psychology testing software (Release 1.5), were used for this experiment.

Trial design. In each session, there were 60 trials, although neither the 1st nor the last 5 were included in subsequent analyses (the first 5 were considered warm-up trials, whereas on the last 5 , the subjects were asked to count aloud). The subjects were instructed that on each trial they would be presented with a series of geometric figures. There were two types of figures, rectangles and triangles. Each figure type appeared in either of two possible orientations. The rectangle $(26 \times 17 \mathrm{~mm})$ was presented with its longer side either on the horizontal or on the vertical. The triangle (base $=$ $26 \mathrm{~mm}$, height $=26 \mathrm{~mm}$ ), with one side horizontal, appeared pointing either up or down. The rectangle subtended a visual angle of $3.7^{\circ} \times 2.4^{\circ}$; the triangle, $3.7^{\circ} \times 3.7^{\circ}$. The subjects were told that the order of presentation of these figures was random. Their task was to keep a count of how many rectangles and how many triangles were presented and to report these counts at the end of each trial. Each trial contained from 16 to 25 figures, presented, one at a time, on a computer screen. It was necessary that the number of figures should vary across trials; if the subjects knew how many figures were to be in a trial, they need only have counted one of the figures, which, when subtracted from a constant total, would yield the other figure count. The subject started a trial by pressing the spacebar, which presented the first figure. Each subsequent barpress cleared the screen of the current figure and presented the next; the response-stimulus interval, during which the next figure was drawn to the screen, varied between 14 and $19 \mathrm{msec}$ (depending on which figure was being presented). The intervals between the presentation of a figure (the clock did not start until the figure was drawn) and the following barpress were recorded. The subjects were told that accuracy was most important but that they should also try to move through each trial as quickly as they could. Feedback, in the form of the correct counts, was presented on the screen after each trial.

There were 6 trials of each trial length ( 16 through 25 figures), thus producing 60 trials. These were ordered randomly. The order of presentation of figures within a trial was also randomly generated, although the trials were screened so as not to allow the successive presentation of the same figure in the same orientation. If, for example, the randomization called for two consecutive rectangles, different orientations were used, the orientation of the first having been randomly chosen. This restriction on the randomization was required so that subjects would receive visual confirmation that a new figure had been presented. This procedure produced trials in which the number of alternations from one type of figure to the other varied, creating the SS and SNS sequences described above.

For the second session, the same 60 trials were presented but in a different random order. Including both sessions, the subjects completed 120 trials, of which 100 were included in the analyses. In total, the subjects were presented with 1,240 rectangles and 1,212 triangles. The order of presentation of these figures produced 1,126 SS and 1,206 SNS, with the first observation of each of the 120 trials being neither.

Procedure. The subjects, run individually, received written instructions on what was required of them for this experiment. The experimenter then performed a demonstration trial. Next, the subjects completed two practice trials. At this point, the subjects donned hearing protectors to reduce extraneous noise, the lights were dimmed, and the experimental session of 60 trials began. On the last 5 trials, the subjects were asked to verbalize their counting.

Except for reading the written instructions and the experimenter's demonstration (subjects completed all three practice trials), the procedure for the second session on Day 2 was identical. 


\section{Results and Discussion}

Error analysis. The data were broken down across sessions (Session 1 vs. Session 2) and within sessions (first half vs. second half of the trials). Each of these four resulting blocks contained 25 trials, with each trial containing 16-25 observations. The number of errors (i.e., incorrect on either the triangle or the rectangle count) was quite high, averaging 7.5 per block of 25 trials (approximately $30 \%$ ). A one-way repeated measures analysis of variance (ANOVA) revealed that the number of errors did not vary across blocks $(F<1)$, although there was considerable variability between subjects $[F(9,30)=$ $6.74, p<.0001$ ], with the mean number of errors per block ranging from 2.25 to 17 . The number of times the subjects got each trial wrong was calculated, yielding a frequency that could vary between 0 and 20 ( 10 subjects performed each trial twice). It was found that the number of times a trial was counted incorrectly was related to the number of figures in that trial $(r=.51)$. This unsurprising finding may be due to the subjects' having been more inclined to make a mistake as more counting was required and as a trial lasted longer. The number of SNSs in a trial appeared to be a better predictor of counting errors than did the number of SSs in a trial $(r \mathrm{~s}=.43$ and .18 , respectively). However, the number of both SNSs and of SSs was also correlated with the number of figures in a trial. Controlling for the effect of the number of figures, the partial correlations between number of SNSs and number of SSs in a trial with the number of times that trial was counted incorrectly were .15 and -.17 , respectively.

The high number of errors might argue against interpreting the RT data. With so many errors, can we be sure that the subjects were diligent in updating their counts and that the RT data are therefore meaningful? A closer look at the subjects' errors helped address these concerns. First, $77 \%$ of the errors were ones in which only one of the counts was incorrect (remember, subjects reported both a rectangle and a triangle count). Of these single-count errors, the correlation coefficient between the incorrect reported count and the actual count was .76. For $74 \%$ of these single-count errors, the incorrectly reported count was just \pm 1 away from the correct count. To summarize, $70 \%$ of all trials were counted correctly, single-count errors of \pm 1 constituted $17 \%$ of the trials, single-count errors of greater than \pm 1 constituted a further $6 \%$, and finally, double-count errors, in which both counts were counted incorrectly, were made on the remaining $7 \%$ of the trials.

An inspection of the distribution of single-count errors revealed a symmetrical distribution of errors, ranging from -6 to 7 (calculated by subtracting the reported count from the true count). The symmetry of errors would suggest no systematic bias (e.g., failures to update the counts would predict underestimation of the correct counts) in making errors.

It would seem reasonable to conclude that although the subjects did make mistakes in their counting, they were not responding with guesses. Though errors were made, it would appear that the subjects were diligent in updating their counts throughout the session, a conclusion supported later when RTs for both correct trials and error trials are compared.

RT analysis. As with the error analysis, the data were broken down across sessions (Session 1 vs. Session 2) and within sessions (first half vs. second half of the trials). A 2 (session) $\times 2$ (half) $\times 2$ (figure type) $\times 2$ (SS/SNS) ANOVA was performed on the subjects' mean RTs from the correct trials only. Note that, unlike in the above error analysis, in which the complete trial was the unit of analysis, we are now looking at means for each subject calculated from the RTs to each individual figure (i.e., the latencies associated with the barpress responses that called up each successive figure during a trial).

From an inspection of the means, a relatively straightforward picture emerges. The subjects were faster on Session 2 than on Session 1, with mean RT dropping from 1,311 to $1,134 \mathrm{msec}[F(1,9)=73.40, p<.0001]$. The subjects were faster during the second half of a session $(1,174 \mathrm{msec})$ than during the first half $(1,271 \mathrm{msec})$ $[F(1,9)=6.77, p=.03]$. Both improvements can plausibly be attributed to a practice effect. The subjects were also faster on SNS than they were on $\operatorname{SS}[F(1,9)=130.39$, $p<.0001]$. Comparing mean SNS and SS RTs provided an estimate of the switching cost. Figure 2 shows the mean RTs for SS and SNS for each subject (for this and for all subsequent graphs, error bars represent the standard error of the mean). The switching costs for the 10 subjects ranged from 306 to $696 \mathrm{msec}$, with a mean of $483 \mathrm{msec}$ (standard deviation [SD] was $144 \mathrm{msec}$ ). A oneway repeated measures ANOVA performed on the switching costs across the four testing blocks (i.e., Session 1, first half; Session 1, second half; Session 2, first half; Session 2, second half) revealed no effect of blocks $[F(3,27)=1.51, p=.23]$.

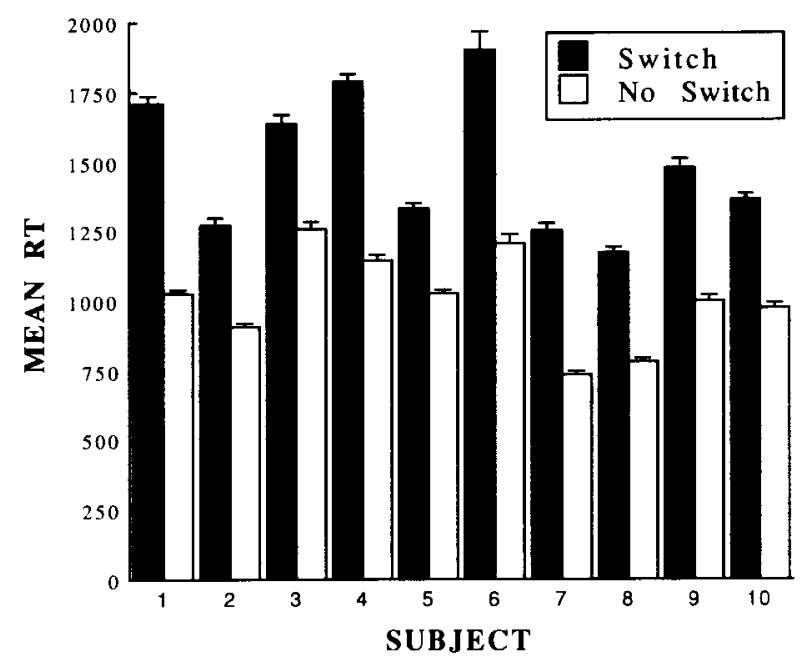

Figure 2. Mean RTs (in milliseconds) for switch/no switch figures for all subjects, Experiment 1. 
As noted above, much of the discarded data (those trials in which the subjects did not report both counts correctly) may still contain meaningful $R T$ data. The above four-way ANOVA was also performed on the RTs from the single-count $( \pm 1)$ error trials, all remaining errors trials, the correct trials combined with the single-count $( \pm 1)$ error trials, and all trials (correct and error alike). In each case, the pattern of results remained unchanged; switching cost estimates, averaged across all subjects, varied from $462 \mathrm{msec}$ to $524 \mathrm{msec}$ for the different mixes of data. Mean switching costs were also calculated for medians (429 $\mathrm{msec})$ and for trimmed RT distributions $(460 \mathrm{msec})$, in which all observations greater than three $S D$ s from the mean were first deleted.

Note that, although the subjects were faster in responding to SNS figures than to SS figures, the magnitudes of the partial correlations between number of SNS and SS figures in a trial and the number of times that trial was counted incorrectly did not suggest an appreciable speed-accuracy trade-off. For each trial, the figure RTs were summed and divided by the number of figures in that trial, yielding a mean trial RT (TRT). Each subject's trials were categorized with respect to whether or not they were correct. As described earlier, four error types were identified: correct, single-count errors of \pm 1 , single-count errors of greater than \pm 1 , and double-count errors. Mean TRTs increased in the order that these error types have been listed, but a one-way repeated measures ANOVA revealed that the rise in TRT was not significant $(F<1)$. This analysis demonstrated that no speedaccuracy trade-off existed on the level of the trial; the subjects were not faster on trials in which they reported incorrect counts. In fact, the mean TRTs were in the direction opposite to what one would expect from a speed-accuracy trade-off.

Counting protocols. All of the subjects, bar one, adopted the same counting technique. These subjects verbalized both counts after the presentation of each figure. For example, if the current counts were five rectangles and seven triangles, the subjects would rehearse this as "five-seven." If the next figure presented was a triangle, the subjects would update the appropriate count and rehearse "five-eight." Similarly, given another triangle, "five-nine," and a rectangle, "six-nine." Note that the verbalization order for these 9 subjects was always rectangle first, triangle second. Presumably, this was because they were asked, at the end of a trial, to report the rectangle count first. As will be discussed later, the adoption of this particular technique was to prove illuminating for revealing the processes involved in internal switches of attention.

\section{EXPERIMENT 2}

Experiment 1 served to establish the experimental task and, in so doing, demonstrated a sizable time cost associated with switching between items in working memory. It is proposed that the existence of this switching cost is a consequence of a fundamental cognitive reality - namely, that we can only attend to one mental object at any one time. Furthermore, attending to more than one object requires switches between these objects. An alternative that remains, however, is that the observed time cost reflects a subject's lack of experience with the task and that, with practice, the effect, and the supposed psychological reality, would disappear. The purpose of Experiment 2 was to give subjects intensive practice, in the hope of reaching asymptotic performance levels on the dual-count task. Intensive practice should also reduce the number of counting errors from that present in Experiment 1.

Experiment 2 also addressed two alternative hypotheses that proposed different priming mechanisms for the switching time costs. The first priming hypothesis suggested a facilitation in the identification of the stimuli. In Experiment 1, one might argue that, for perceptual reasons alone, the time to identify a figure may have been affected by the preceding figure. Rectangles and triangles each have features not shared by the other. It might be argued that a recent activation and integration of the features of one figure may facilitate subsequent activation and integration of those same features. Having just seen a triangle, it may be easier to identify a second triangle. Similarly, having just seen a rectangle, it may be easier to identify a second rectangle. This possible facilitatory effect would thus predict that SNS responses would be faster than SS responses. Thus, Experiment 2 used two squares as the two stimuli, one small (approximately $14 \times$ $14 \mathrm{~mm}$ ) and one large (approximately $26 \times 26 \mathrm{~mm}$ ).

Nevertheless, the use of two squares that have identical features, save size, may minimize but does not completely eliminate this possible confound. The large and small squares are still two distinct percepts, and perceiving one figure may yet facilitate the subsequent perception of that same figure. Consequently, a more direct test of facilitation in the stimulus identification stage was deemed necessary.

A second priming hypothesis concerns facilitation between repeated activations, not of the perceptions, but rather of the count representation itself. Perhaps, having just updated one count (e.g., the one for large squares) that count or, more precisely, its internal representation may remain active for some time. If another large square is presented before the count representation has decayed to baseline activation levels, there may be a facilitation in accessing and subsequently performing operations on that count. No such facilitation would be present if a small square was presented. Thus, this conceptual-priming hypothesis, which is similar to the hypothesis of an internal attentional limitation, predicts faster responses for SNS than for SS. Note that, although the two alternatives make the same prediction, they do differ in what are proposed as the underlying mechanisms that produce the switching cost. The internal attention hypothesis proposes that SS RTs are longer than SNS RTs because a limited capacity attentional focus must be shifted, an act 
that is time consuming. The conceptual-priming hypothesis, on the other hand, proposes that SNS RTs are faster, without reference to any internal attention focus. Instead, SNS RTs are presumed faster as the activation of one count persists above baseline when reactivated for another update. The dual-count task was modified, as described below, to test the conceptual-priming alternative.

\section{Method}

Subjects. The subjects were five new students drawn from an introductory psychology subject pool. There were no special criteria for inclusion in this study. The experiment required at least 14 sessions, each $30 \mathrm{~min}$ to $1 \mathrm{~h}$ long, held at the same time of day on 14 consecutive days. The subjects received credit in partial fulfillment of their course requirements and payment for their participation.

Apparatus. A Macintosh SE computer and SuperLab, a general purpose psychology testing software (Release 1.5), were used for this experiment.

Trial design. In each session there were 60 trials. The number of squares in each trial varied between 16 and 25 . The order of presentation of squares within a trial was randomly generated. This procedure produced trials in which the number of alternations from one square to the other varied. A square that was different from the immediately preceding square was coded as an SS, whereas a square that was the same as the immediately preceding one was coded as an SNS. The letter $\mathrm{X}$ was presented between each square in order to signal to subjects that a new square had been presented. The duration of the $\mathrm{X}$ was varied, as described below. The duration of the X was not included in the subjects' RTs; the timer began once the new figure was drawn on the screen. At the end of each trial, the subjects reported the number of each type of square presented in that trial. Feedback, in the form of the correct counts, was presented on the screen after each trial.

A set of 120 trials ( 12 trials of each trial length) was created. For each session, a random set of 60 trials was selected from this set of 120 , thus ensuring that the subjects were presented with a different set of trials in a different order in each session. One consequence of using a fixed set of trials is that, throughout the 14 days of testing, the subjects completed the same trial, on average, seven times. However, the possibility that a subject might learn to recognize trials, thus circumventing the need to maintain two running counts in later sessions, was deemed negligible. The subjects had no reason to suspect that trials would be repeated, and trials did not occur at predictable locations within a session or even in predictable sessions. Instead, the seven repetitions were randomly scattered throughout the 14 sessions. Also, in earlier sessions, the possibility of incidentally learning a random series of, on average, 20 large and small squares while maintaining two running counts was assumed to be very unlikely.

Including all sessions, the subjects completed 840 trials, of which 770 were included for analyses (the first 5 trials in each session were considered warm-up trials). Totaling across all 14 sessions, the subjects were presented, on average, with 8,603 large squares and 8,581 small squares. The order of presentation of these squares produced, on the average, 7,922 SS and 8,432 SNS, with the first observation of each of the 840 trials being neither. Two subjects volunteered to complete extra sessions; Subject 4 completed 15 sessions (8,539 SS and 9,072 SNS), and Subject 3 completed 18 $(10,158 \mathrm{SS}$ and $10,810 \mathrm{SNS})$. These subjects received additional payment for their continued participation.

Stimulus identification priming. To determine whether there is facilitative priming in the stimulus identification stage, the subjects completed a stimulus identification task at the end of each session. The stimuli for this task were the two squares from the dual-count task. Each trial of this task contained a fixation point, a prime figure, a second fixation point, a target figure, a response, feedback, and a delay before the next trial. After a 200 -msec fixation point (the same small $X$ that was used in the dual-count task), one square (the prime) was displayed for $500 \mathrm{msec}$, and the subjects were instructed not to respond to it. The fixation point was then presented again for a variable duration ( $55 \mathrm{msec}, 305 \mathrm{msec}$, and $555 \mathrm{msec}$ ). The reasons for varying these interstimulus intervals (ISIs) are given below. Next, a second square (the target) was presented, and the subject was instructed to respond to it as quickly as possible without sacrificing accuracy. The target remained on-screen until the subject responded. The task was to identify the second square as either large or small. These responses were made with two separate keys, the $m$ and the $n$ keys (the mapping between key and response was counterbalanced across subjects). After the subject's response, the correct response was displayed for $200 \mathrm{msec}$. After a 1,000-msec delay, a new trial started.

The stimuli allowed for four prime-target permutations, two congruent (large-large and small-small), and two incongruent (large-small and small-large). Combining the four permutations with the three different delays produced 12 distinct trial types. The stimulus identification task contained 60 trials, 5 of each trial type. These 60 trials were presented in a different random order for each session and for each subject. The 60 trials were preceded by 12 similar practice trials ( 1 of each trial type) that were not included in analyses. These 12 practice trials were also presented in a different random order for each session and for each subject. The total number of trials (72) were presented in two blocks of 36 trials. The subjects could rest during the interval between blocks and could initiate the second block when they were ready.

The perceptual-priming explanation should predict faster responses on the congruent permutations, relative to the incongruent permutations. That there should be no difference between the two permutation types would argue against a perceptual-priming effect.

Count representation priming. The conceptual-priming hypothesis proposes that a count's representation may remain active for a period of time, facilitating subsequent activation. As noted, this hypothesis and the attention-switching hypothesis make the same prediction for SS and SNS RTs. However, one difference between the two proposed processes is that priming should be sensitive to the intervals between the presentation of the two figures. Therefore, different response-stimulus intervals (RSIs) were introduced between successive figures in the dual-count task. One of five delays $(55,180,305,430$, and $555 \mathrm{msec})$ was randomly selected for each RSI. RSI varied within subjects. If priming underlay the switching costs, SNS RTs should be affected by these RSIs. A priming effect was assumed to predict maximum facilitation at the shortest interval. As the intervals increased-that is, as the activation level of the count representation returned to baseline-the amount of facilitation should decrease. Thus, as the intervals increased, SNS RTs should get slower and the switching cost smaller. Following the same logic, intervals were also added to the stimulus identification task. Just three ISIs were included in order to maximize the number of observations per interval.

In summary, the priming manipulations were designed to reveal whether a priming process was present in the dual-count task, whether such priming was due to facilitation in identifying the stimuli and/or in accessing and updating the count representations, over what intervals priming might play a role, and, consequently, at what intervals an uncontaminated measure of an internal attentionswitching cost might be observed.

Procedure. The instructions to the subjects were similar to those for Experiment 1, except that these subjects were told that they would be presented with a series of large and small squares. As in Experiment 1, the subjects were told that accuracy was most important but that they should also try to move through each trial as quickly as they could. For all sessions, the subjects wore hearing protectors in order to reduce extraneous noise, and the lights were dimmed. Each day, before starting the session of 60 trials, the sub- 


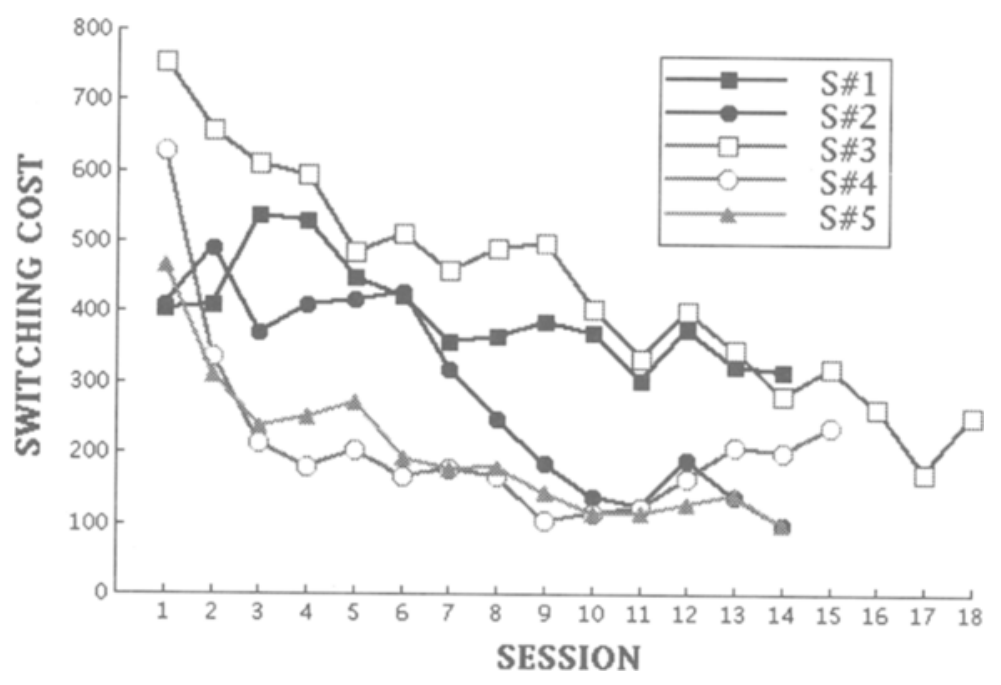

Figure 3. Individual switching costs on each session, Experiment 2.

jects also completed 3 additional practice trials. Approximately every 4 th day, the subjects were told how accurate they had been on the previous day's session, and, if their error rate was high (more than 5 trials in which either count was incorrect), they were encouraged to reach a higher accuracy level. To gain access to their counting strategies, the subjects were asked to perform the same task while counting aloud on 3 additional trials at the end of their first, seventh, and final sessions. These additional trials were not included in the quantitative analyses. On each day, the subjects completed the stimulus identification task last.

\section{Results and Discussion}

Dual-count task analyses. Overall, the error rate was low, averaging $7.2 \%$. The correlation between session (the first 14 sessions only) and the number of errors, summed over subjects, was $r=-.25$. Only correct trials were analyzed.

All RT distributions were first trimmed by discarding RTs greater than three $S D$ s from the mean. Only trimmed distributions were analyzed. Although the subjects did improve noticeably over the 14 sessions, a switching cost was always present (see Figure 3). Across all sessions, the smallest switching costs for Subjects 1 to 5 were $302 \mathrm{msec}$, $100 \mathrm{msec}, 172 \mathrm{msec}, 105 \mathrm{msec}$, and $98 \mathrm{msec}$, respectively. It is, however, unclear whether all of the subjects had reached asymptotic performance. Subjects 2 and 3, in fact, appeared still to have been improving. Nonetheless, within the confines of this experiment, one may conclude that switching costs resistant to practice (i.e., greater than zero) were obtained. This evidence is consistent with a model of internal attention in which one is limited to an internal focus large enough for just one object.

A 2 (SNS/SS) $\times 5$ (RSIs) $\times 14$ (sessions) repeated measures ANOVA was performed on the mean RTs for the 5 subjects. Only data from the first 14 days were included for those subjects who completed more days. The ANOVA revealed that all main effects produced significant differences. The effects of session $[F(13,52)=35.89, p<$
$.0001]$ and switch $[F(1,4)=32.79, p=.005]$ have already been described. Figure 4 presents the SS and SNS RTs as well as the switching cost averaged across subjects for the first 14 sessions (note that session and switch
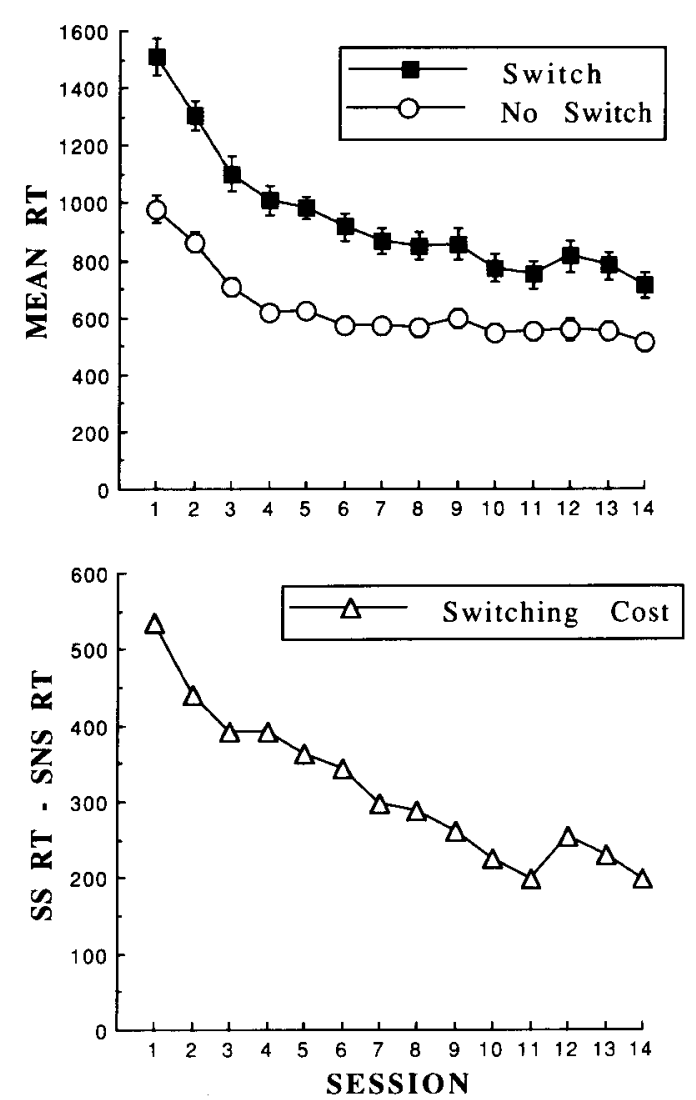

Figure 4. Group mean stimulus switch, stimulus no switch, and switching cost, averaged across sessions and subjects, Experiment 2. 


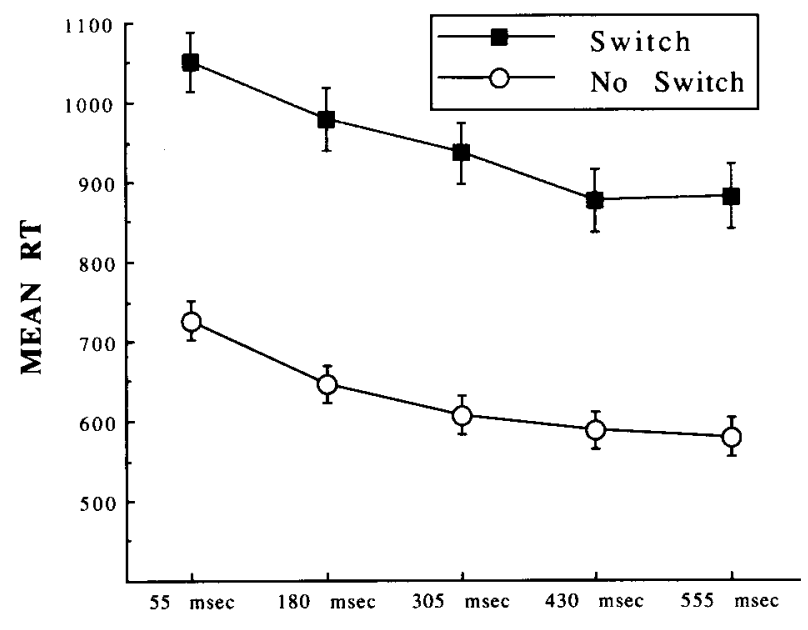

RESPONSE-STIMULUS INTERVALS

Figure 5. Stimulus switch and stimulus no switch RTs for each response-stimulus interval, Experiment 2.

accounted for $30 \%$ and $25 \%$ of the variance, respectively). An inspection of this figure also suggests that the interaction between session and switch $[F(13,52)=$ $9.94, p<.0001$ ] may be attributed to the subjects' reaching near asymptotic performance on SNS trials early, while showing a slower improvement on SS trials.

The intervals, or RSIs, between successive presentations of the figures also had a significant effect $[F(4,16)=$ 29.76, $p<.0001$ ], while accounting for $4 \%$ of the variance. Figure 5 shows the effect of the intervals on both SNS and SS RTs. The decrease in SNS RT as the intervals increased is not consistent with a priming process. The conceptual-priming hypothesis should predict that SNS RTs would get slower and the switching cost smaller as the intervals increased. The SS/SNS $\times$ RSIs interaction is significant $[F(4,16)=5.75, p<.005]$, but accounts for just $0.1 \%$ of the variance. The SS trials, given that they require the subject to update a new count, may not be the ideal control condition for comparison of a priming effect in the SNS trials. However, the absence of a substantial interaction effect suggests that whatever recovery process was responsible for the subjects' responding faster as the intervals increased was common to both SS and SNS and was, therefore, not obscuring a hidden priming effect in SNS. The three-way interaction SS/SNS $\times$ RSIs $\times$ sessions was not significant $(F<1)$.

Individual 2 (SNS/SS) $\times 2$ (large/small squares) $\times 5$ (RSIs) $\times 14$ (sessions) ANOVAs were also calculated for each subject. All sessions were included for those subjects who completed more than 14 days. The observations in each cell of this ANOVA are the subjects' raw RT data (averaging 49 RT data points per cell). Given that the population to which this statistic generalizes is the individual across time, all four factors of these ANOVAs were treated as between subject. The results of these analyses support the conclusions drawn from the group analyses. For all subjects the effects of session, switch, and delay were significant (all $p \mathrm{~s}<.001)$. Unlike the case in Experiment 1, the order in which the counts were reported at the end of each trial was counterbalanced across subjects. This was expected to determine the order in which the subjects verbalized both counts during a trial. This met with mixed success. Three subjects verbalized their counts in the order large square count first, small square count second; 1 subject adopted the opposite order (small square count, large square count); the last subject varied the order, letting the first figure of each trial be the first one verbalized. No clear effect for verbalization order was found. For the 3 subjects for whom a main effect for size was significant, RTs to the small square were faster. A perceptual explanation for this finding is not supported by the stimulus identification task (presented below), in which a difference in speed of responding to the squares was not found.

The individual ANOVAs had great statistical power (for the SS/SNS factor, all $p \mathrm{~s}<1.0 \times 10^{-30}$ ), and most interactions were significant although effect sizes were small. Only one such significant interaction, switch $X$ session, which has already been discussed for the grouped data, accounted for more than $1 \%$ of the variance and did so for 4 of the 5 subjects. No interesting interpretations of the other interactions were evident. Finally, Table 1 presents the switching cost and individual, lower $99.9 \%$ confidence levels for each subject's last session.

Counting protocols. As previously described for Experiment 1 , when asked to count aloud, all of the subjects adopted the technique of verbalizing both counts after each figure - that is, the subjects would update one count and rehearse the current value of the other count. For convenience, these different operations will be referred to as updating and rehearsing. For these speakaloud trials, the subjects were asked to count as they had during the session's trials. They were not given instructions to count in any particular manner, so as to allow them freedom to arrive at their own preferred technique. Presumably it is safe to assume that they employed the updating and rehearsing technique, subvocally, on the dual-count task. This counting technique is also similar to that reported retrospectively by the subjects in the running count tasks of Monty and his colleagues (Monty, Taub, \& Laughery, 1965; Monty, Wiggins, \& Karsh, 1969).

Adopting this counting technique produces the following interesting circumstance. Imagine a subject has two counts, $A$ and $B$, one for each of two types of fig-

Table 1

Switching Costs (in Milliseconds) and the Lower 99.9\% Confidence Level for Each Subject's Last Session

\begin{tabular}{ccc}
\hline Subject & $\begin{array}{c}\text { Switching Cost } \\
\text { (SS - SNS) }\end{array}$ & $\begin{array}{c}\text { Confidence Level } \\
99.9 \%\end{array}$ \\
\hline 1 & 316 & 266 \\
2 & 100 & 48 \\
3 & 254 & 201 \\
4 & 237 & 179 \\
5 & 98 & 67 \\
\hline
\end{tabular}


ures, $a$ and $b$. The subject rehearses these counts in the order "A-B," which I refer to as the verbalization order. When a figure $a$ is presented, the subject subvocally updates the A count and then subvocally rehearses the B count in the fixed order, "A-B." Note what has happened here. The A count, having just been updated, is assumed to be in the focus of attention, and yet the B count was most recently rehearsed. Imagine that a $b$ is presented next. Though $B$ is the count that was most recently rehearsed, there is still a cost incurred in switching from the A count to the B count. To demonstrate this, one can compare the size of the switching cost for the first and the second counts of each subject's verbalization order. Of interest is determining if there is still a sizable switching cost when switching to the second count of the verbalization order, having updated the first (i.e., switching to the count that one has most recently rehearsed). Let us call this a Type 1 switch. The complement to this is switching to the first count in the verbalization order, having just updated the second count (i.e., switching to the count that one has not most recently rehearsed). This is denoted a Type 2 switch.

To demonstrate whether Type 1 and Type 2 switches were equally large, the size of the former can be expressed as a percentage of the latter ( $100 \%$ would thus mean that the two types of switches were equally long). The mean RT for Type 1 switches, as a percentage of the mean RT for Type 2 switches, was calculated for Subjects 1, 3, 4, and 5 (the percentage was not calculated for Subject 2, who did not have a fixed verbalization order). Looking at the data from each subject's last session only, the percentages are $101 \%, 113 \%, 96 \%$, and $89 \%$, for Subjects $1,3,4$, and 5, respectively. For Subjects 1 and 3, Type 1 switches were slower than Type 2 switches; for Subjects 4 and 5 , this pattern was reversed. What these percentages reveal is a sizable Type 1 switching cost - that is, a sizable switching cost even when one is switching to the count that one has most recently rehearsed. The implications of this finding will be treated in the General Discussion.

Stimulus identification analyses. The subjects' accuracy on this task was very high, with errors on just $1 \%$ of the trials, averaged across 14 sessions for the 5 subjects. Only correct responses were analyzed. A 2 (congruent/incongruent) $\times 2$ (large/small squares) $\times 3$ (ISIs) $\times 14$ (sessions) ANOVA was calculated for the subjects' mean RTs. Only data from the first 14 days were included for those subjects who completed more days.

A marginally significant effect was found for the congruent/incongruent factor $[F(1,4)=5.93, p=.07]$, but it was in the opposite direction to that predicted by a priming explanation; subjects were faster at making the large/small judgement for the incongruent permutations, as compared to the congruent permutations (see Figure 6). The subjects did improve over sessions $[F(13,52)=$ $7.39, p<.0001]$, but there was no interaction between sessions and the congruent/incongruent factor $(F<1)$. As indicated above, no statistical difference was found

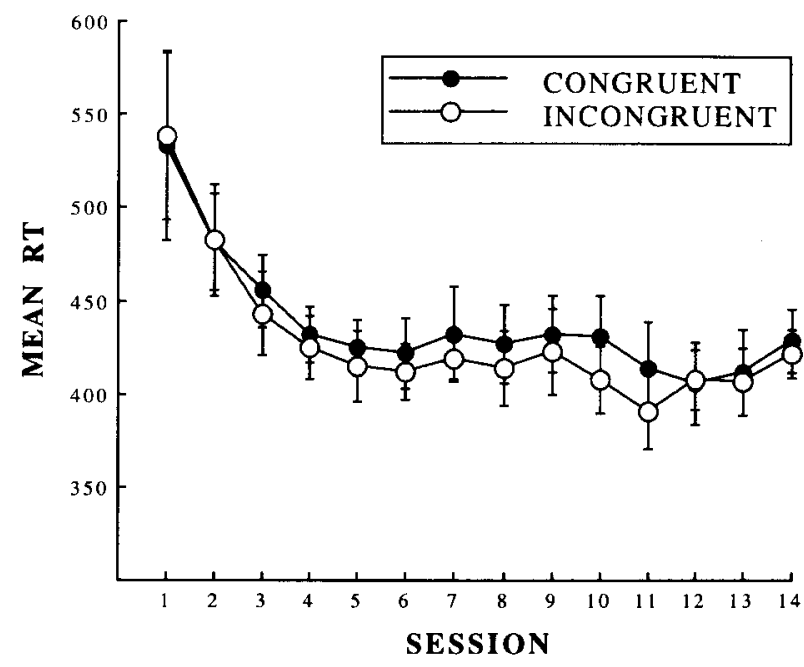

Figure 6. Mean RTs (in milliseconds) for congruent and incongruent trials from the stimulus identification task, Experiment 2.

for the size factor $(F<1)$. Finally, there was an effect for the ISIs in this task $[F(2,8)=66.9, p<.0001]$, but it was in the opposite direction to that predicted by a priming explanation, with the subjects responding faster as the intervals increased. Mean RTs for the 55-, 305-, and 555 -msec intervals were 464,426 , and 410 msec, respectively. The increase in response speed held equally for both congruent and incongruent permutations, with no evidence of an interaction present $(F<1)$.

\section{GENERAL DISCUSSION}

This study has shown empirically that subjects cannot access two counts in working memory with equal speed. Instead, with this particular task, the count most recently updated can subsequently be updated faster. If the count that was not most recently updated must be accessed, a time cost is incurred. This time cost is estimated at $483 \mathrm{msec}$ early in practice (Experiment 1) and drops, after intensive practice, to between $98 \mathrm{msec}$ and $316 \mathrm{msec}$ across subjects (Experiment 2).

At a theoretical level, it is proposed that these time costs serve as estimates for the operation of an attentionswitching mechanism. The attention-switching mechanism operates between the count representations resident in working memory, with internal attention focused on just one count representation at a time. That the time costs remain constant across the imposed response-stimulus intervals suggests that, for this task, internal attention does not move until there is reason to switch, internal attention switching being initiated by the presentation of the stimulus for the other count. The existence of an internal attention-switching cost reveals a difference in the accessibility of the two working memory counts and strongly suggests that mental objects such as counts are processed serially. Consequently, one might conclude that 
the existence of a switching cost is incompatible with information-processing models that allow for the simultaneous focusing of attention on more than one working memory item.

The results also argue against possible priming hypotheses. In the stimulus identification task, a subject's response to a figure was not facilitated when that figure was preceded by an identical figure. Instead, the marginally significant trend was toward faster responses to a figure that was preceded by a different figure. This suggests that any perceptual advantage to be had would have resulted in an underestimation of switching times. Within the dual-count task, the effect of introducing delays between the figures also ran contrary to a repetitionpriming hypothesis; SNS RTs did not get slower, and the switching cost did not get smaller, as the intervals between figures increased. The data also suggest that what emerged to be a facilitatory effect of increased intervals did not obscure a priming effect. Finally, one may presume that the scope of a priming explanation is limited to smaller switching costs and cannot explain the large switching costs found early in practice; indeed, even some highly practiced subjects finished with switching costs that might be considered too large to suggest a priming phenomenon.

\section{A Simple Process Model}

Figure 7 outlines a simple model of the processes proposed as being involved in the dual-count task. Having identified the stimulus, the subjects must orientate their attention to the appropriate count, update that count, rehearse the current value of the other count, and, finally, make a response in order to call up the next stimulus. It is the second process, the orientation of attention, that

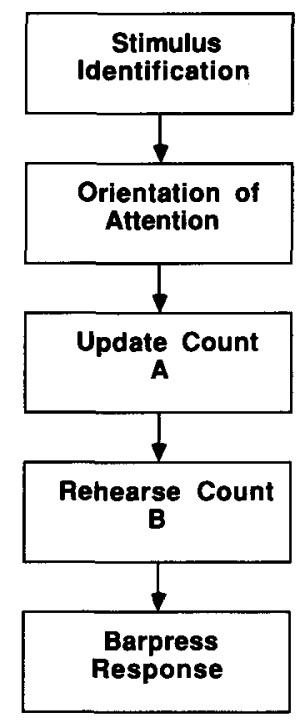

Figure 7. A model of the processes involved in performance of the dual-count task. The order of the update and rehearse operations is dictated by which figure is presented and by the subject's verbalization order. has been the subject of this paper. Following an SS, attention must be reoriented from one count to the other, and this process takes time. For an SNS, in which the same count is successively updated, no such reorientation is required. The order of the updating and rehearsing operations is determined both by which figure is being presented and by a subject's verbalization order. Note that the orientation of attention precedes the updating and rehearsing operations. Note also that at no point were the subjects required to perform two operations simultaneously, nor were they required to make or reconfigure different responses. Instead, the existence of a central attentional limitation was inferred by manipulating the order in which the counts were to be attended.

Clearly, the experiments reported rely on a subtractive method for estimating the cost involved in the reorientation of attention. An assumption of pure insertion inevitably accompanies this method. Certain characteristics of the dual-count task may alleviate concerns that this assumption is violated (or, at least, reduce the degree to which it is violated). The stimulus identification task found that any differences in identifying one figure as a function of the preceding figure would, if anything, serve to underestimate the switching cost. A trend suggested that a figure different from the preceding figure was identified faster than a figure the same as the preceding figure. In the dual-count task, the updating and the rehearsing operations occurred after each figure - that is, both operations were performed, and both counts were subvocally verbalized, irrespective of whether a switch of attention had occurred. The verbalization order also remained constant within a trial and was not altered by whether a switch of attention had occurred. Finally, the same simple response, a barpress, was also required on all trials. Nevertheless, it may be the case, for example, that the updating step in the sequence of processes may be faster if one does not have to switch to the count being updated. Though this must remain a possibility, it was not the case that repeated, successive updates of the same count quickened the updating operation (i.e., time to update a count did not get faster as a function of the number of successive updates of that same count).

Nonetheless, it is an inescapable fact that other processes can influence the size of the switching cost. It is evident that individual differences and practice are two such influences. Others include the duration of the trial (switching costs were higher as the counts increased) and even the difference in the current values of the two counts. For these reasons, a pure measure of the switching process may always be elusive. Because of both the inevitable uncertainties that accompany use of the subtraction logic and the existence of extraneous influences on task performance, I would rather place emphasis, not on the precise value of the estimated switching cost parameter, but on the more defensible observation that the parameter estimate is not zero. It is this observation that affords the conclusion of an attentional limitation within working memory. 
This said, it is notable that, even after intensive practice, the observed switching costs were quite large. The memory search literature, which is most relevant to the present study, typically reveal smaller switching costs. Schneider and Shiffrin (1977) estimated both a switching cost of $42 \mathrm{msec}$ and a switching cost of $27 \mathrm{msec}$ from the data of Briggs and Johnsen (1973). Sternberg (1966) found that each new item in the positive set added $40 \mathrm{msec}$ to the search task (according to Sternberg's model, the $40 \mathrm{msec}$ included both a comparison process and a switching cost). Introducing the requirement to also report the location of the item in the memory set increased the searching time to $250 \mathrm{msec}$ per item.

It may be the case that the difficulty of the operation being performed on the items between which one is switching determines the size of the switching cost. Scanning a memory set to detect a match to a target item seems, intuitively, to be a simpler task than updating the value of a count. In the search paradigm, the matching working memory item, once identified, receives no further processing. The complexity of the operation may require more resources of a limited resource pool, leaving fewer available resources for control operations such as attention switching. This was the argument employed by Laabs and Stager (1976) in their binaural listening task to explain why switching from serial addition (presumably the more difficult task) to shadowing produced greater interference than did switching in the other direction. In a test of switching between perceived lists and memorized lists, Weber et al. (1986) noted that longer lists produced longer switching times, which is also compatible with the notion of straining limited resources.

\section{Consequences of an Internal Attention \\ Limitation for Working Memory}

The counting technique adopted by all of the subjects (except 1 subject from Experiment 1) proved informative. Throughout each trial, the subjects maintained two running counts, both of which were subvocally verbalized after each new figure was presented. The Type 1 switch proved to be as long as the Type 2 switch - that is, a switch to the count that one had most recently rehearsed was as time demanding as a switch to the count that one had not most recently rehearsed. Both the existence of a switching cost and the finding that Type 1 switches were as large as Type 2 switches provide us with a number of insights into the relationship between working memory and the focus of internal attention.

\section{Attention Switching and Memory Retrieval}

The cost associated with switching between items in working memory does not appear to be due to memory retrieval. The count being switched to in the Type 1 switch was the most recently rehearsed count, and, therefore, one would expect the retrieval of that count's current value to be at least as fast as, if not faster than, retrieval of the just updated count. Thus, accomplishing switches between items in working memory requires a distinct mechanism, which I have described in terms of a switch of attentional focus, rather than being due to a memory retrieval operation. Logically, one may also question the plausibility of a retrieval-based explanation for the switching costs observed in the dual-count task. Clearly, both counts are already in working memory; therefore, one must ask of a retrieval-based explanation, into what are the counts being retrieved? To push the retrieval explanation to its eventual conclusion, it would seem that such a theory should state that one of the two counts in working memory must be retrieved into a subset of working memory, a subset of less than two items. This model would appear to be identical to the internal attention explanation proposed herein. Given that both counts are already present in working memory, the attentional focus may provide the more coherent description.

\section{Internal Attention and Working Memory Status}

One might assume that working memory items are all equal. For example, the linear functions of the search paradigm tasks reveal that each additional item in the memory set adds a fixed amount of time to the searching process. But items in working memory do not have equal status, as is evidenced in the present study by the fact that, although both items are verbalized after each update, there is a distinct difference in how quickly either can be subsequently updated. To reiterate, the item in the focus of attention can be updated faster, which is inconsistent with the assumption that items in working memory have equal accessibility. Further insights into the nature of this differential status could be gleaned by increasing the number of items. With just two counts, the present study cannot reveal whether there is a qualitative distinction between the item in the focus of attention and all other items (which would enjoy equal status) or whether there is a continuum in the status of working memory items. The status of an item in working memory may be determined by how recently it was last updated or by how many other intervening items have been subsequently updated. A search for such patterns in the data from the present study revealed no such effects. For example, the cost incurred in switching to a count was not affected by how recently that count had last been updated (that is, the time to switch to one count was not affected by the number of intervening updates of the other count).

One may wish to describe the status of the items in working memory in terms of their activation levels. The object in the focus of attention presumably has the highest activation level. For an item to be in the focus of attention might require that it be maintained at this relatively high activation level. Once attention is taken away from that item, its activation returns to the baseline level required to keep the item within working memory. The evidence from the present study suggests a fast return to these baseline levels. Recall that the priming manipulations found no facilitation effects that would indicate a decaying activation function that has residual effects. 
Similarly, as noted earlier, other unreported, in-depth analyses have shown that varying the number of repeated activations of one item did not affect the speed of subsequent responses to that item (i.e., time to update a count did not get faster as a function of the number of successive updates of that same count). Nor, indeed, did repeated activations (updates) of one count hinder access to the other count (as mentioned above, the time to switch to a count was not affected by the number of immediately preceding updates of the other count). Combined, these patterns suggest that a step function is involved in being in or out of the focus of attention.

A third consequence that arises from the Type 1 switching cost is that items can be rehearsed (though not updated) without the focus of attention being drawn to them. The focus of attention remains on one count, even after the current value of the other count has been rehearsed. In one sense, then, an item in working memory can be rehearsed, which requires some degree of processing, without becoming the object of thought. This finding might best be accommodated under a multiplecomponents theory of working memory, such as Baddeley's (Baddeley \& Hitch, 1974).

\section{Internal Attention Switching and the Phonological Loop}

While performing the dual-count task, both counts may reside in a verbal rehearsal buffer akin to the phonological loop. This loop has been described as a subsystem of working memory, a "phonological store that relies on a fading trace which can be maintained by subvocal rehearsal" (Baddeley, 1986, p. 81). This subsystem, although linked, can operate somewhat independently of the functioning of the central executive, which is responsible for various control processes. Many tasks involving learning and comprehension can be accomplished with a far from catastrophic decrement in performance, even with a concurrent digit span task (see Baddeley, 1986, chapters 3 and 4). In the dual-count task, both counts may reside in such a verbal buffer while attention, controlled by the central executive, is switched between them. Thus, the rehearsal operation and the focus of attention operate independently. Morris and Jones (1990) arrived at a similar explanation for their running memory task. Their subjects were asked to recall a prespecified number of the most recent items from a list of consonants that were presented serially. As list length could not be predicted, this task required that the subjects update the items being rehearsed once the list exceeded the number to be recalled. Both the number of updates of the list and performing secondary tasks known to disrupt the function of the phonological loop impaired recall. However, there was no interaction between number of updates and the presence or absence of the secondary tasks, suggesting that the updating operation was accomplished by the central executive.
Although earlier versions of Baddeley's model did ascribe storage capacity to the central executive (Baddeley, 1976), subsequent versions have not (Baddeley, 1993); hence, both counts are considered to reside in the phonological loop rather than, say, one count in the loop and one "in" the central executive. This raises the interesting issue of determining whether the limitation in attending to more than one object at a time is specific to a single subsystem. To put it another way, could one have immediate and simultaneous access to both a verbal working memory item and a visual working memory item? It remains to be demonstrated whether the internal limitation that has been demonstrated by the dual-count task reflects a limitation specific to one subsystem of working memory or whether it is a limitation that traverses subsystems.

\section{Some Functional Consequences of an Internal Attention Limitation}

If there is a limitation in the number of working memory items on which one can simultaneously focus, then what functional consequences might this have? In his classic 1956 paper, Miller emphasized the importance of chunking given the $7 \pm 2$ limitation on the number of items that can be held in working memory. Through chunking, we afford ourselves access to more than just $7 \pm 2$ elementary items of information. By increasing the size of these chunks, we can increase the amount of information contained in working memory. One can consider the formation of these chunks of information as a strategic or adaptive response that accommodates this working memory limitation. If so, it is possible that chunking is equally likely to have been a strategic response to another working memory limitation - namely, that which is the focus of this paper. If we can only focus our attention within working memory on one item at a time, it is surely beneficial to be able to attend to more than a single bit of information. ${ }^{1}$

A limitation in attending to working memory items, if true for counts, should also be true for more complex items, such as scientific hypotheses or theories. Although it runs counter to the exhortations of some scientists and philosophers of science (see, e.g., Platt, 1964; Popper, 1962), an abundance of evidence demonstrating a neglect of alternative hypotheses has been garnered from laboratory investigations of hypothesis-testing behaviors (Mynatt, Doherty, \& Tweney, 1977, 1978). This tendency can affect both the information that one looks for, as in Wason's four-card task (Wason \& Johnson-Laird, 1972), and one's interpretation of new information that bears on the truth or falsity of one's original hypothesis, as in the pseudodiagnosticity research (Doherty, Mynatt,Tweney, \& Schiavo, 1979; Kern \& Doherty, 1982; Mynatt, Doherty, \& Dragan, 1993). While the dualcount task employed in this study is certainly far removed from the complexity and richness of hypothesis 
testing in human inference, the present study nevertheless proposes that the internal attention limitation is a fundamental one. Consequently, the same limitation would also operate in an inference task and may partially explain the lack of selection or production of data relevant to alternative hypotheses.

\section{Conclusion}

As previously noted, some early psychologists asserted that it was phenomenologically self-evident that we can attend to just one object at a time. The present study attempted to empirically demonstrate this limitation, explored what such a limitation might reveal about working memory function, and gave examples of what implications might follow from such a limitation. In a recent commentary on the state of STM research, Shiffrin makes the point that attention and memory research "make it clear that attentional focus cannot be identified with the entire set of currently activated information, but represents a far smaller subset instead" (Shiffrin, 1993, p. 195). The present study has demonstrated, at least for verbal information such as running counts, that this subset contains just one item. If we can attend to but one object at a time, we are obliged to switch between objects. This study has measured and described the dynamics of this switching. In so doing, it concludes that a distinct attention-switching mechanism is involved; it has attempted to rule out visual repetition priming, conceptual repetition priming, and memory retrieval mechanisms as alternative explanations.

\section{REFERENCES}

BadDELEY, A. D. (1976). The psychology of memory. New York: Basic Books.

BADDELEY, A. D. (1986). Working memory. Oxford: Oxford University Press, Clarendon Press.

BADDELEY, A. D. (1993). Working memory or working attention. In A. D. Baddeley \& L. Weiskrantz (Eds.), Attention: Selection, awareness, and control. A tribute to Donald Broadbent (pp. 152-170). Oxford: Oxford University Press.

BadDELEY, A. D., \& ECOB, J. R. (1973). Reaction time and short-term memory: Implications of repetition effects for the high-speed exhaustive scan hypothesis. Quarterly Journal of Experimental Psychology, 25, 229-240.

BadDeley, A. D., \& Hitch, G. (1974). Working memory. In G. H. Bower (Ed.), The psychology of learning and motivation (Vol. 8, pp. 47-90). New York: Academic Press.

BRIGGS, G. E. (1974). On the predictor variable for choice reaction time. Memory \& Cognition, 2, 575-580.

BRIGGS, G. E., \& JoHNSEN, A. M. (1973). On the nature of central processing in choice reactions. Memory \& Cognition, 1, 91-100.

Carlson, R. A., Wenger, J. L., \& Sullivan, M. A. (1993). Coordinating information from perception and working memory. Journal of Experimental Psychology: Human Perception \& Performance, 19, 531-548.

Corballis, M. C., Kirby, J., \& Miller, A. (1972). Access to elements of a memorized list. Journal of Experimental Psychology, 94, 185190.

COWAN, N. (1988). Evolving conceptions of memory storage, selective attention, and their mutual constraints within the human informationprocessing system. Psychological Bulletin, 104, 163-191.

CoWAN, N. (1993). Activation, attention, and short-term memory. Memory \& Cognition, 21, 162-167.
DARK, V. J. (1990). Switching between memory and perception: Moving attention or memory retrieval? Memory \& Cognition, 18, 119 127.

Doherty, M. E., Mynatt, C. R., Tweney, R. D., \& Schiavo, M. D. (1979). Pseudodiagnosticity. Acta Psychologica, 43, 111-121.

GreENe, R. L. (1992). Human memory: Paradigms and paradoxes. Hillsdale, NJ: Erlbaum.

GuZY, L. T., \& AXELROD, S. (1972). Interaural attention shifting as response. Journal of Experimental Psychology, 95, 290-294.

JAMES, W. (1890). The principles of psychology. New York: Henry Holt.

JERSILD, A. T. (1927). Mental set and shift. Archives of Psychology, 14 (Whole No. 89).

Kern, L., \& Doherty, M. E. (1982). "Pseudodiagnosticity" in an idealized medical problem-solving environment. Journal of Medical Education, 57, 100-104.

Kerr, B. (1973). Processing demands during mental operations. Memory \& Cognition, 1, 401-412.

LAABS, G. J., \& STAGER, P. (1976). Monitoring the information processing demands of attention switching. Canadian Journal of Psychology, 30, 47-54.

Mewhort, D. J. K., Thio, H., \& Birkenmayer, A. C. (1971). Processing capacity and switching attention in dichotic listening. Canadian Journal of Psychology, 25, 111-129.

MiLLER, G. A. (1956). The magical number seven, plus or minus two: Some limits on our capacity for processing information. Psychological Review, 63, 81-97.

Monty, R. A., TAUB, H. A., \& LAUGHERY, K. R. (1965). Keeping track of sequential events: Effects of rate, categories, and trial length. Journal of Experimental Psychology, 69, 224-229.

MonTY, R. A., WigGins, H. F., \& Karsh, R. (1969). Keeping track of sequential events: Manipulation of the incrementing process. Journal of Experimental Psychology, 80, 408-411.

MORRIS, N., \& JONES, D. M. (1990). Memory updating in working memory: The role of the central executive. British Journal of Psychology, 81, 111-121.

Mynatt, C. R., DoherTy, M. E., \& Dragan, W. (1993). Information relevance, working memory, and the consideration of alternatives. Quarterly Journal of Experimental Psychology, 46A, 759-778.

MynatT, C. R., DoherTy, M. E., \& TWeney, R. D. (1977). Confirmation bias in a simulated research environment: An experimental study of scientific inference. Quarterly Journal of Experimental Psychology, 29, 85-95.

Mynatt, C. R., Doherty, M. E., \& Tweney, R. D. (1978). Consequences of confirmation and disconfirmation in a simulated research environment. Quarterly Journal of Experimental Psychology, 30, 395-406.

Pillsbury, W. B. (1973). Attention. New York: Macmillan. (Original work published 1908)

Platt, J. R. (1964). Strong inference. Science, 146, 347-353.

POPPER, K. R. (1962). Conjectures and refutations. New York: Basic Books.

RatCliff, R. (1978). A theory of memory retrieval. Psychological Review, 85, 59-108.

Rogers, R. D., \& Monsell, S. (1995). Costs of a predictable switch between simple cognitive tasks. Journal of Experimental Psychology: General, 124, 207-231.

SCHNEIDER, W., \& SHIFFrin, R. M. (1977). Controlled and automatic human information processing: I. Detection, search, and attention. Psychological Review, 84, 1-66.

SHIFFRIN, R. M. (1993). Short-term memory: A brief commentary. Memory \& Cognition, 21, 193-197.

Shiffrin, R. M., \& SCHneIDER, W. (1977). Controlled and automatic human information processing: II. Perceptual learning, automatic attending, and a general theory. Psychological Review, 84, 127-190.

SPECTOR, A., \& BIEDERMAN, I. (1976). Mental set and mental set revisited. American Journal of Psychology, 89, 669-679.

STERNBERG, S. (1966). High-speed scanning in human memory. Science, 153, 652-654.

STERNBERG, S. (1967). Two operations in character recognition: Some evidence from reaction-time measurements. Perception \& Psychophysics, 2, 45-53. 
STERNBERG, S. (1969). Memory scanning: Mental processes revealed by reaction-time processes. American Scientist, 57, 421-457.

TEN HOOPEN, G., \& Vos, J. (1981). Attention switching and patterns of sound locations in counting clicks. Journal of Experimental Psychology: Human Perception \& Performance, 7, 342-355.

Theors, J., Smith, P. G., Haviland, S. E., Traupmann, J., \& Moy, M. C. (1973). Memory scanning as a serial self-terminating process. Journal of Experimental Psychology, 97, 323-336.

WASON, P. C., \& JoHNSON-LAIRD, P. N. (1972). Psychology of reasoning: Structure and content. Cambridge, MA: Harvard University Press.
Weber, R. J., BuRt, D. B., \& Noll, N. C. (1986). Attention switching between perception and memory. Memory \& Cognition, 14, 238-245.

\section{NOTE}

1. I thank Elke Kurz for conveying this point to me.

(Manuscript received July 15, 1996; revision accepted for publication December 27, 1996.) 